\title{
Circulating chemerin levels and gestational diabetes mellitus: a systematic review and meta-analysis
}

\author{
Zhongwei Zhou ${ }^{\dagger}$, Hongmei Chen ${ }^{\dagger}$, Huixiang Ju and Mingzhong Sun ${ }^{*}$
}

\begin{abstract}
Background: Chemerin is a novel adipokine which is associated with metabolic syndrome and type 2 diabetes mellitus. However, recent investigations regarding circulating chemerin levels in gestational diabetes mellitus (GDM) are conflicting. This meta-analysis is to evaluate and determine their associations.

Methods: A systematic literature search was performed in PubMed, EMBASE and Web of Science up to 13 December 2017. Pooled standardized mean differences (SMD) and 95\% confidence interval (Cl) were calculated using a random-effect model.

Results: Eleven studies comprising 742 GDM patients and 840 normal pregnant women were included. Circulating chemerin levels were increased in GDM patients compared with healthy pregnant women (SMD: 1.16; 95\% Cl: 0.29, 2. $04 ; P=0.009$ ). Subgroup analyses revealed such difference was especially available in the groups of the second trimester (SMD: 1.47; 95\% Cl: 0.28, 2.67) and mean age < 30 years (SMD: 2.30; 95\% Cl: 0.69, 3.91) of GDM patients. There was significant heterogeneity among studies $\left(P^{2}=98.0 \%, P<0.001\right)$; however, heterogeneity disappeared or markedly decreased in the subgroups of European populations $\left(P^{2}=0.0 \%, P=0.531\right)$, age $\geq 30$ years $\left(P^{2}=28.2 \%, P=0.223\right)$ and WHO diagnostic criteria $\left(P^{2}=0.0 \%, P=0.490\right)$ when stratifying by study location, trimester of chemerin measurement and the diagnostic criteria of GDM.
\end{abstract}

Conclusions: The elevated levels of circulating chemerin were associated with GDM, which suggests it might play an important role in the pathogenetic mechanism of GDM.

Keywords: Chemerin, Gestational diabetes mellitus, Insulin resistance, Systemic inflammation, Meta-analysis

\section{Background}

Gestational diabetes mellitus (GDM) is defined as varying degrees of glucose intolerance first detected during pregnancy, which affects $4-18 \%$ pregnant women according to different diagnostic criteria and ethnic origin $[1,2]$. The pathophysiologic mechanism of GDM is similar to type 2 diabetes mellitus (T2DM), including insulin resistance, oxidative stress and systemic inflammation [3]. It is also suggested that pregnant women who develop GDM may have pre-existing $\beta$-cell defects unable to adapt to the increasing demands of insulin during pregnancy $[4,5]$, and now it is widely believed that

\footnotetext{
* Correspondence: 15189300678@163.com

${ }^{\dagger}$ Zhongwei Zhou and Hongmei Chen contributed equally to this work. Department of Clinical Laboratory, Affiliated Yancheng Hospital, School of Medicine, Southeast UniversityJiangsu 224001 Yancheng, People's Republic of China
}

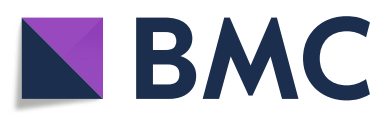

(อ) The Author(s). 2018 Open Access This article is distributed under the terms of the Creative Commons Attribution 4.0 International License (http://creativecommons.org/licenses/by/4.0/), which permits unrestricted use, distribution, and reproduction in any medium, provided you give appropriate credit to the original author(s) and the source, provide a link to the Creative Commons license, and indicate if changes were made. The Creative Commons Public Domain Dedication waiver (http://creativecommons.org/publicdomain/zero/1.0/) applies to the data made available in this article, unless otherwise stated. and the subsequent insulin resistance in diabetic patients $[6,7]$. So, whatever view one takes, it is undeniable that insulin resistance and chronic low-grade inflammation play vital roles in the progression of GDM.

Chemerin is a novel cytokine mainly secreted from white adipose tissues, which was initially considered as a chemotactic factor generated in inflammatory conditions, but more recently, it was reported more as an adipokine regulating metabolism of adipose and balance of energy $[8,9]$. Serum levels of chemerin were shown to be markedly elevated in patients with biopsy-proven non-alcoholic fatty liver disease (NAFLD) compared with healthy controls [10], and elevated hepatic chemerin mRNA expression also was confirmed to be independently associated with liver fibrosis, steatosis, 
inflammation, and hepatocyte ballooning in human NAFLD [11]. A recent population-based study revealed that increased chemerin levels were associated with inflammation and metabolic syndrome even after adjustment for waist circumference [12]. More importantly, chemerin has been indicated to be an independent predictor of type 2 diabetes mellitus (T2DM) and cardiovascular event risk [13, 14]. Recent studies also suggested that chemerin may play an important role in the pathogenetic mechanism of GDM. However, studies of the association between circulating chemerin levels and GDM yielded inconsistent findings. Therefore, we carried out a meta-analysis to provide a more comprehensive estimation of the association between circulating chemerin levels and GDM.

\section{Methods}

\section{Search strategy}

A systematic literature search was carried out in electronic databases including PubMed, EMBASE and Web of Science up to 13 December 2017. The search terms included: chemerin AND ("gestational diabetes mellitus" OR "gestational diabetes" OR "GDM"). In addition, the references from these relevant articles were manually searched for additional eligible studies.

\section{Inclusion and exclusion criteria}

Original research evaluating the associations between circulating chemerin levels and pregnancy outcomes were considered eligible if they met the following criteria: (1) GDM as outcome and the control were healthy pregnant women with normal glucose tolerance (NGT); (2) all the subjects did not have a previous history of diabetes or present pregnant complications; or (3) studies were published in English or Chinese. Studies were excluded if they were letters to the editor, short report, conference abstracts, reviews, or studies on animals or cell lines.

\section{Data extraction}

Two investigators (Zhongwei Zhou and Hongmei Chen) independently reviewed all identified studies and extracted the data using a predefined form, and confirmed by a third reviewer (Mingzhong Sun). Disagreement was resolved by discussion among all researchers. The following information was abstracted from each eligible study: the first author's name, year of publication, study location, study design, trimester of chemerin measurement, average age and body mass index (BMI) of GDM patients, diagnostic criteria of GDM, sample size of the case and control group, mean and standard deviation (SD) of chemerin.

\section{Quality assessment}

The quality of the study was evaluated using a modified criteria based on the Newcastle-Ottawa Quality Assessment Scale (NOS) for observational studies suggested by van Dijk et al. [15], The full score was 9 stars, and a study that met 7 or more stars would be considered as a high-quality study, less than 3 stars low-quality study, and other studies were defined as moderate quality.

\section{Statistical analysis}

Standardized mean differences (SMD) and 95\% confidence interval (CI) was calculated and estimated the differences in circulating chemerin levels between GDM patients and controls. A random-effect model which is more conservative than the fixed effect model was chosen for pooling of data [16]. Heterogeneity among studies was assessed using Cochran's Q-test at $p<0.1$, and quantified by the $I^{2}$ index, and an $I^{2}$ index of 25, 50 and $75 \%$ would indicate small, moderate and high heterogeneity, respectively [17]. Subgroup analysis was carried out to explore possible explanations for heterogeneity. Sensitivity analysis was performed to valuate the influence of a single on the pooled measures by omitting one study in each turn and recalculating the pooled SMD for the remainders. Publication bias was evaluated by inspection of funnel plots, and the Egger's test. All analyses were performed using Stata14.0 (StataCorp LP, College Station, TX, USA) and $P<0.05$ was considered to be statistically significant.

\section{Results}

\section{Literature search}

A flowchart of the included and excluded studies is showed in Fig. 1. A total of 55 records were identified after an initial search from the selected electronic databases. After removing duplicates and reading the titles and abstracts, 15 appropriate articles were identified for full text scrutiny. We further excluded 3 studies for lack of necessary data, and one study not in English or Chinese. Finally, 11 studies (13 results) met the criteria and were selected for the final analysis [18-28].

\section{Characteristics of the included studies}

The 11 included studies were published from 2010 to 2017 covering 742 GDM patients and 840 normal pregnant women. The characteristics of the studies included in the present are presented in Table 1. Of the 11 included studies, all of them were cross-sectional, and ELISA methods were applied to measure circulating chemerin levels in all the studies. Five studies were carried out in China, two in Turkey, one in Pakistan, one in Australia, one in Germany and one in the Kingdom of Saudi Arabia (KSA). Seven studies measured circulating chemerin levels during the second trimester, five 


\section{5 potentially relevant articles identified through datebase searching \\ 13 PubMed \\ 21 EMBASE \\ $21 \mathrm{Web}$ of Science}

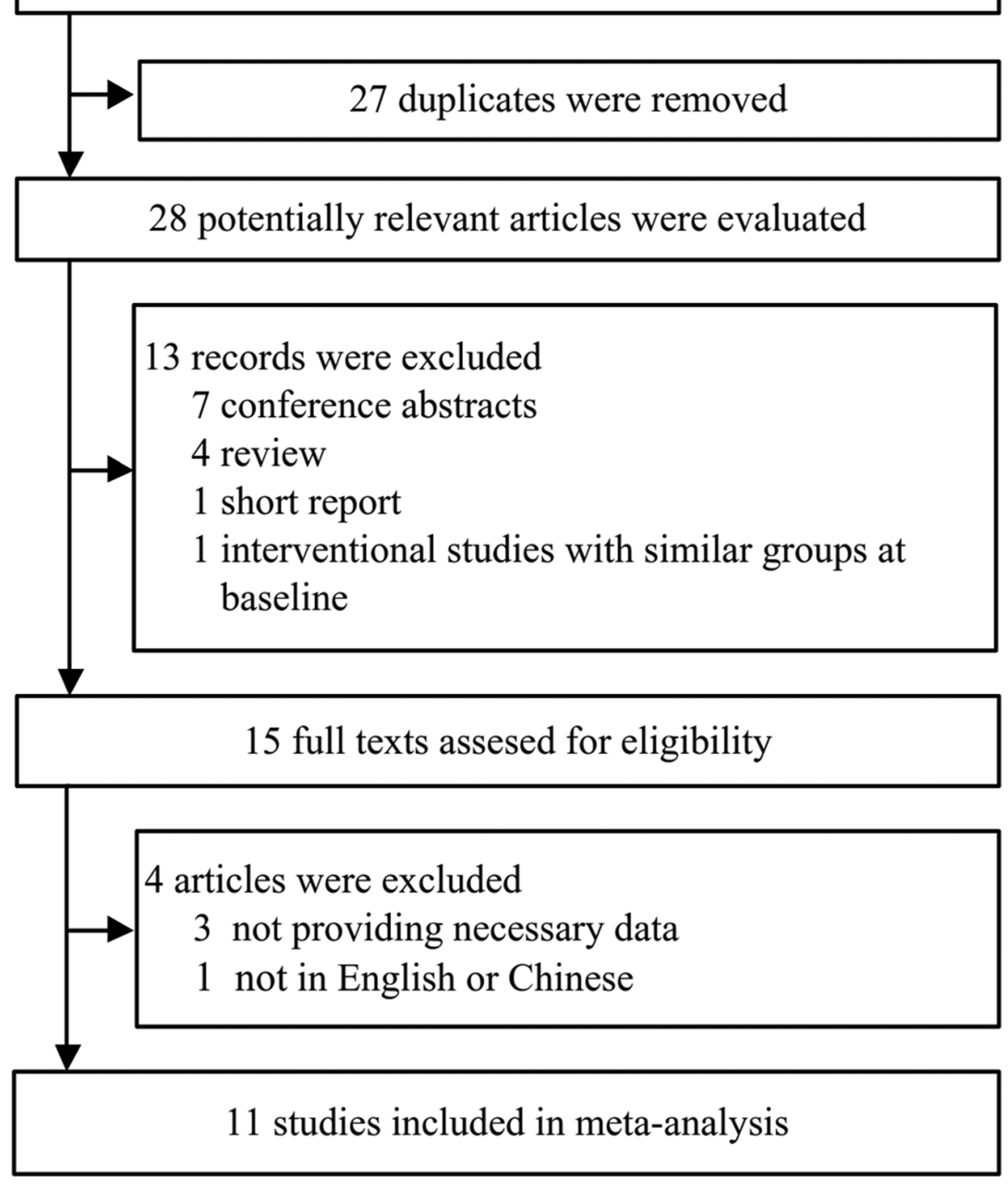

Fig. 1 Flow chart of the study selection process

during the third trimester and one during the first trimester. GDM was diagnosed based on several different criteria, and among them, Fourth International Work-shopVconference on gestational diabetes (WC), The Australasian Diabetes in Pregnancy Society (ADIPS), The International Association of Diabetes and Pregnancy Study Groups (IADPSG), American Diabetes Association (ADA), Carpenter and Couston (C\&C), Endocrine Society Clinical Practice Guideline (ESCPG),
American College of Obstetricians and Gynecologists (ACOG) and World Health Organization (WHO) were used in different studies. The sample size of these studies ranged from 19 to 208 among GDM patients, and 20 to 300 among normal pregnant women. The range of mean chemerin levels was 1.65 to $308.6 \mathrm{ng} / \mathrm{mL}$ among GDM patients, and 1.57 to $227.5 \mathrm{ng} / \mathrm{mL}$ among normal pregnant women. The quality assessment results showed that two studies $[20,28]$ were assessed as 


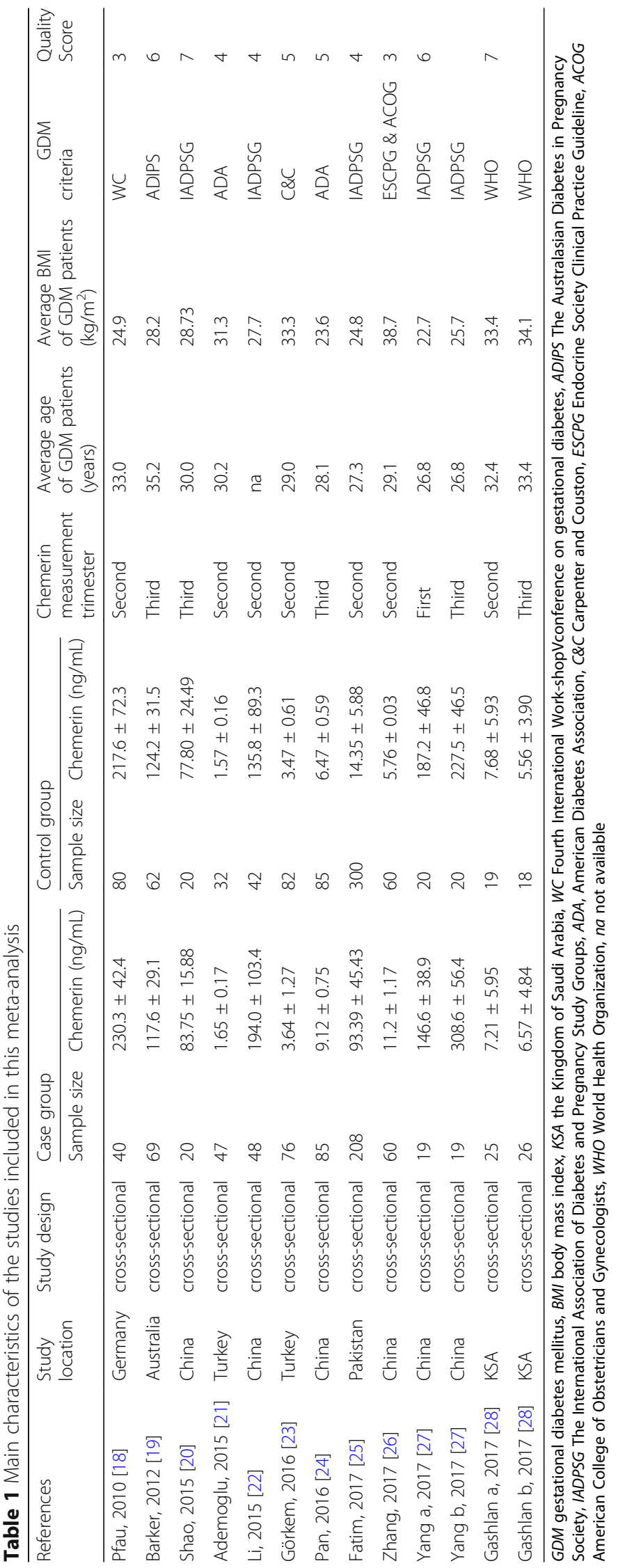


high quality, and the other nine studies were all assessed as moderate quality, which was exhibited in Table 1.

\section{Overall meta-analysis}

We performed a random-effects meta-analysis on the extracted 11 studies. As indicated in Fig. 2, the overall levels of circulating chemerin in GDM patients were significantly increased (SMD: 1.16; 95\% CI: 0.29, 2.04; $P=$ $0.009)$ when compared with healthy pregnant women. Sensitivity analysis showed that no individual study had a significant effect on the circulating chemerin levels between GDM patients and healthy pregnant women. No significant publication bias was found in this meta-analysis by Egger's test $(P=0.324)$, and the funnel plot with pseudo $95 \%$ confidence limits is shown in Fig. 3. However, significant heterogeneity among studies was found in this meta-analysis $\left(I^{2}=98.0 \%, P<0.001\right)$.

\section{Subgroup analyses}

To explore the potential source of the high levels of heterogeneity among studies, we performed subgroup analysis according to study location, trimester of chemerin measurement, the average age and BMI of the GDM patients and the diagnostic criteria of GDM. When stratifying by study location, these studies were classified as Asian group, European group and Oceanian group. As shown in Fig. 4, both the Asian group (SMD: 1.63; 95\% CI: $0.42,2.84$ ) and the European group (SMD: 0.25; $95 \%$ CI: $0.03,0.46)$ showed higher circulating chemerin levels among women with GDM, especially in the Asia; and although no heterogeneity was observed in the European group $\left(I^{2}=0.0 \%, P=0.531\right)$, considerable heterogeneity were still found in the Asian group $\left(I^{2}=98.0 \%, P<\right.$ 0.001 ). When stratifying by trimester of chemerin measurement, the second-trimester patients showed significantly higher levels of circulating chemerin compared with healthy pregnant women (SMD: 1.47 ; 95\% CI: 0.28, 2.67), while the difference did not reach statistical significance during the third trimester (SMD: 1.15; 95\% CI: $-0.46,2.76)$, and GDM patients in the first trimester showed even significantly decreased chemerin levels than those in healthy pregnant women (SMD: -0.92 ; 95\% CI: $-1.59,-0.26)$; however, significant heterogeneity among studies was still observed in the second group $\left(I^{2}=98.4 \%, P<0.001\right)$ and the third trimester group $\left(I^{2}=97.8 \%, P<0.001\right)$ (Fig. 5). In the subgroup analysis of the average age, GDM patients with mean age $<30$ years had significantly increased circulating chemerin levels when compared with controls (SMD: 2.30; 95\% CI: 0.69, 3.91); however, the difference was not significant between patients with mean age $\geq 30$ years and controls (SMD: 0.12; 95\% CI: $-0.11,0.35$ ); significantly decreased heterogeneity was observed in the group of age $\geq 30$ years $\left(I^{2}=28.2 \%, P=0.223\right)$, but not in the group of age $<30$ years $\left(I^{2}=98.7 \%, P<0.001\right)$ (Fig. 6). In stratified analyses based on mean BMI of GDM patients, the difference of the chemerin levels between the GDM patients and controls was more significant in the group of mean BMI < 28 (SMD: 1.34; 95\% CI: 0.02, 2.66) than

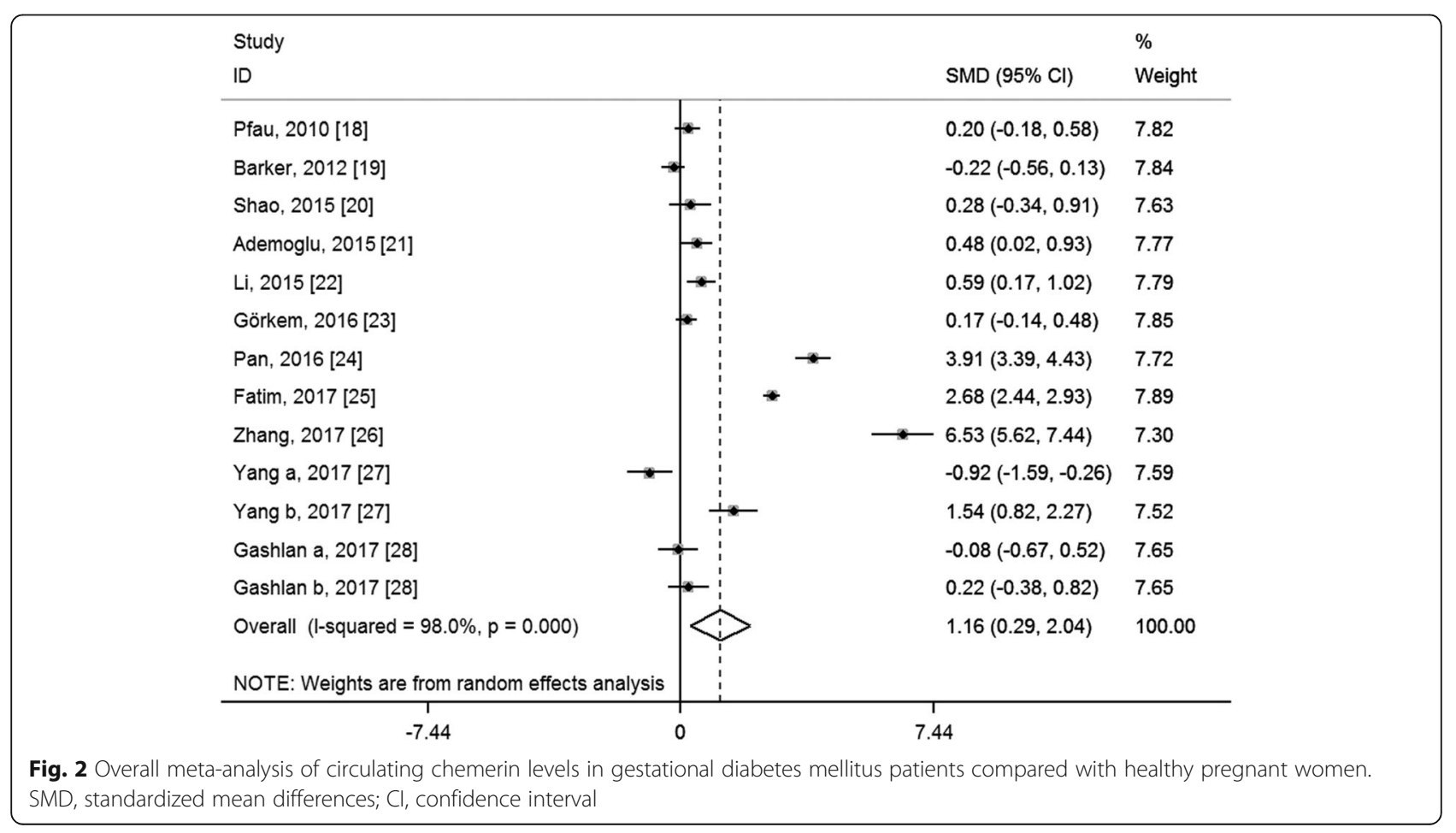




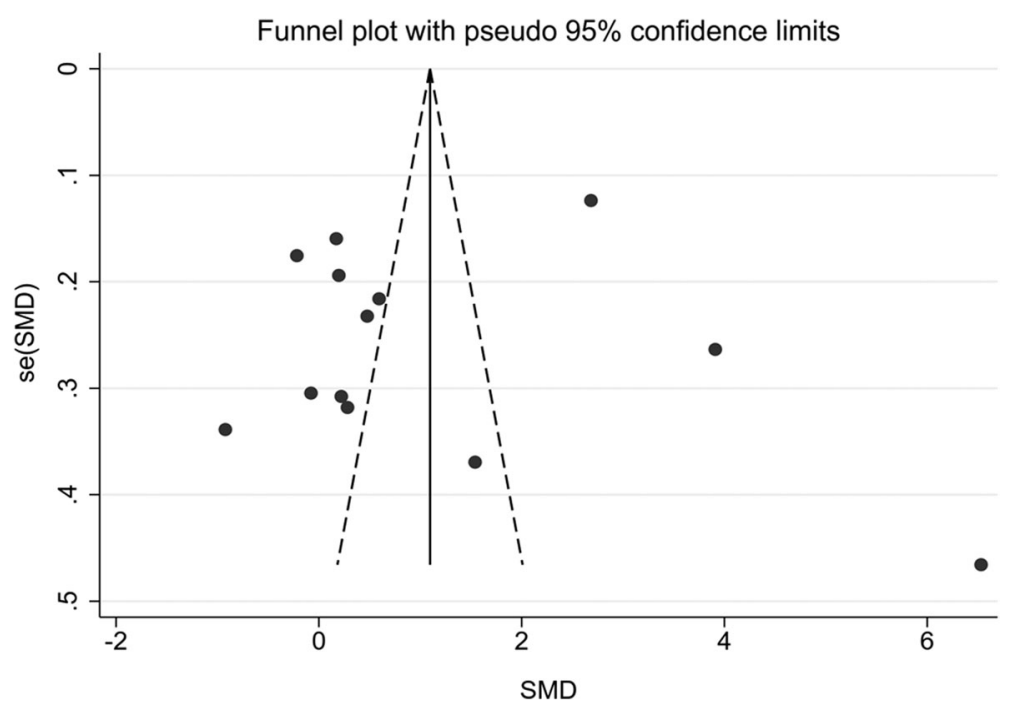

Fig. 3 Funnel plot of included studies for potential publication bias between gestational diabetes mellitus patients and healthy pregnant women. SMD, standardized mean differences; se, standard error

that of mean BMI $\geq 28$ (SMD: 1.00; 95\% CI: $-0.03,2.03$ ), but dramatic heterogeneity were still observed in both groups $\left(I^{2}=98.2\right.$ and $96.8 \%$ respectively; $\left.P<0.001\right)$ (Fig. 7). When stratifying by the diagnostic criteria of GDM, patients defined by ESCPG \& ACOG criteria had significantly higher chemerin levels compared with control (SMD: 6.53; 95\% CI: 5.62, 7.44), but those defined by other criteria had not; although no heterogeneity was observed in the WHO subgroup $\left(I^{2}=0.0 \%, P=\right.$ $0.490)$, significant heterogeneity were still found in the IADPSG subgroup $\left(I^{2}=97.6 \%, P<0.001\right)$ and ADA subgroup $\left(I^{2}=99.0 \%, P<0.001\right)$ (Fig. 8 ).

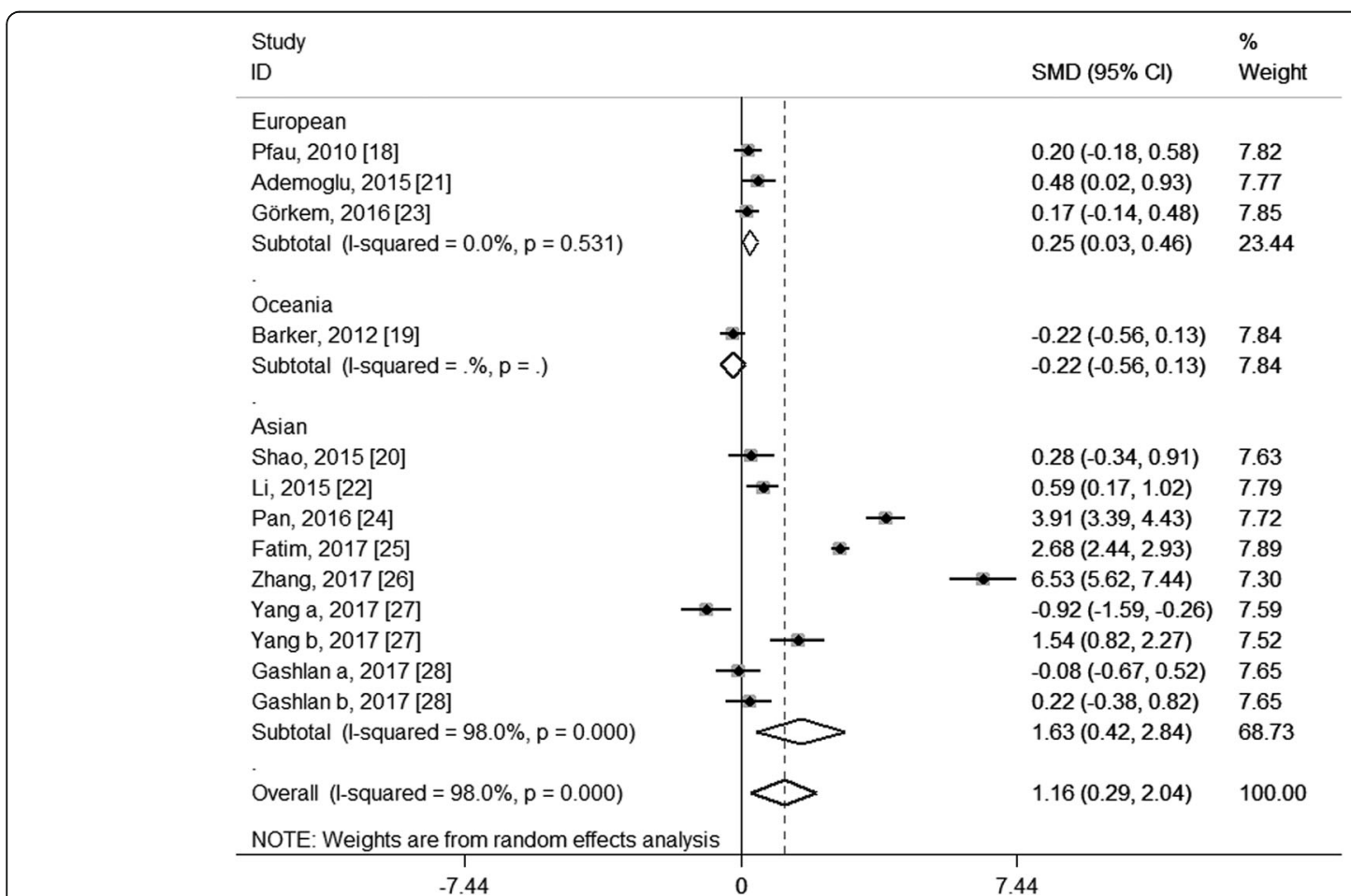

Fig. 4 Subgroup analysis of circulating chemerin levels in gestational diabetes mellitus patients compared with healthy pregnant women when stratified by study location. SMD, standardized mean differences; Cl, confidence interval 


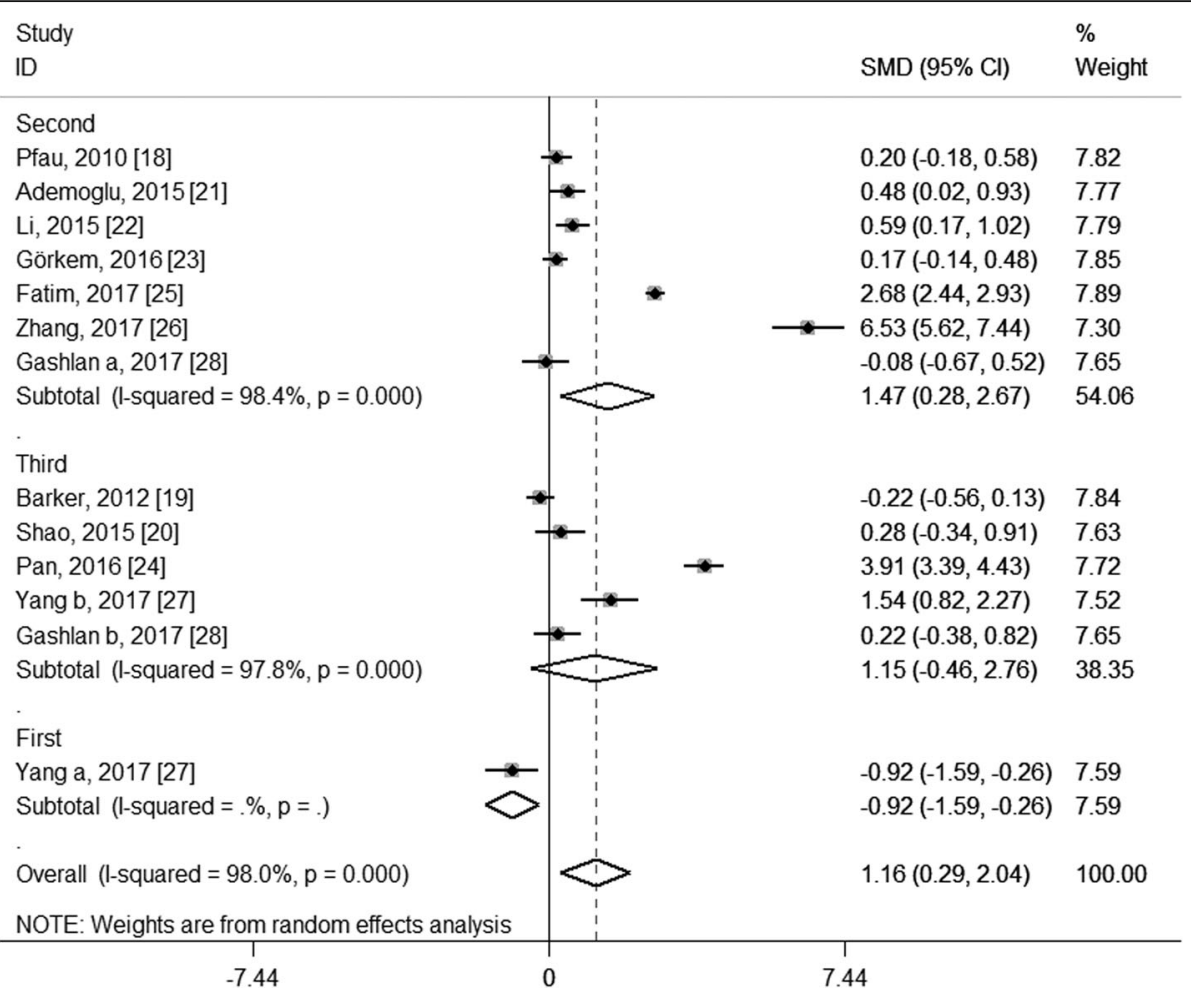

Fig. 5 Subgroup analysis of circulating chemerin levels in gestational diabetes mellitus patients compared with healthy pregnant women when stratified by trimester of chemerin measurement. SMD, standardized mean differences; $\mathrm{Cl}$, confidence interval

\section{Discussion}

This meta-analysis demonstrated that the circulating chemerin levels was significantly increased in women with GDM than healthy pregnant women. Sensitivity analysis showed that the pooled results were not unduly influenced by a particular study. In addition, we did not find significant publication bias in this meta-analysis. To the best of our knowledge, this is the first meta-analysis on this subject, which suggests chemerin may play an important role in the pathogenetic mechanism of GDM.

In the meta-analysis, subgroup analysis was used to analyze the potential factors contributing to heterogeneity and obtain further information from different sub-populations. When stratifying by study location, average age of the GDM patients and the diagnostic criteria of GDM, we found heterogeneity disappeared or markedly decreased in the subgroups of European populations, age $\geq 30$ years and WHO diagnostic criteria, which suggests study location, age of GDM patients and the diagnostic criteria of GDM may be associated with heterogeneity at some level. In addition, we found the significant difference of circulating chemerin levels between GDM patients and healthy pregnant women was especially available in the subgroups of the second trimester but not the third trimester. In line with the pooled results, one studies [28] included in the present meta-analysis demonstrated that both GDM patients and healthy pregnant women had higher chemerin levels in the second trimester than those of corresponding groups in the third trimester. Recent research indicated that adipokines such as chemerin are released from human serum albumin, apart from human adipose tissue [29]; and serum albumin levels are usually decreased in late pregnancy because of increasing nutrition needs of fetus, which may partly account for the lower levels of circulating chemerin for GDM patients during the third trimester measurement. But this phenomenon that the significant difference of chemerin levels was observed in the second-trimester patients may be the result of more studies included in the subgroup. Therefore, further large-scale investigations are needed to confirm this result and expound its significance. We also observed the significant difference of the comparison of GDM vs controls in the subgroups of mean age $<30$ years rather than $\geq 30$ years, suggesting that there may be a negative correlation between age and circulating chemerin levels in GDM patients. However, there is little research involved in the relationship of age with chemerin in women with GDM. Two recent studies reported that there were weakly negative correlations between age and serum chemerin levels in T2DM patients, but which was not significant [30, 31]. Thus more research is also needed 


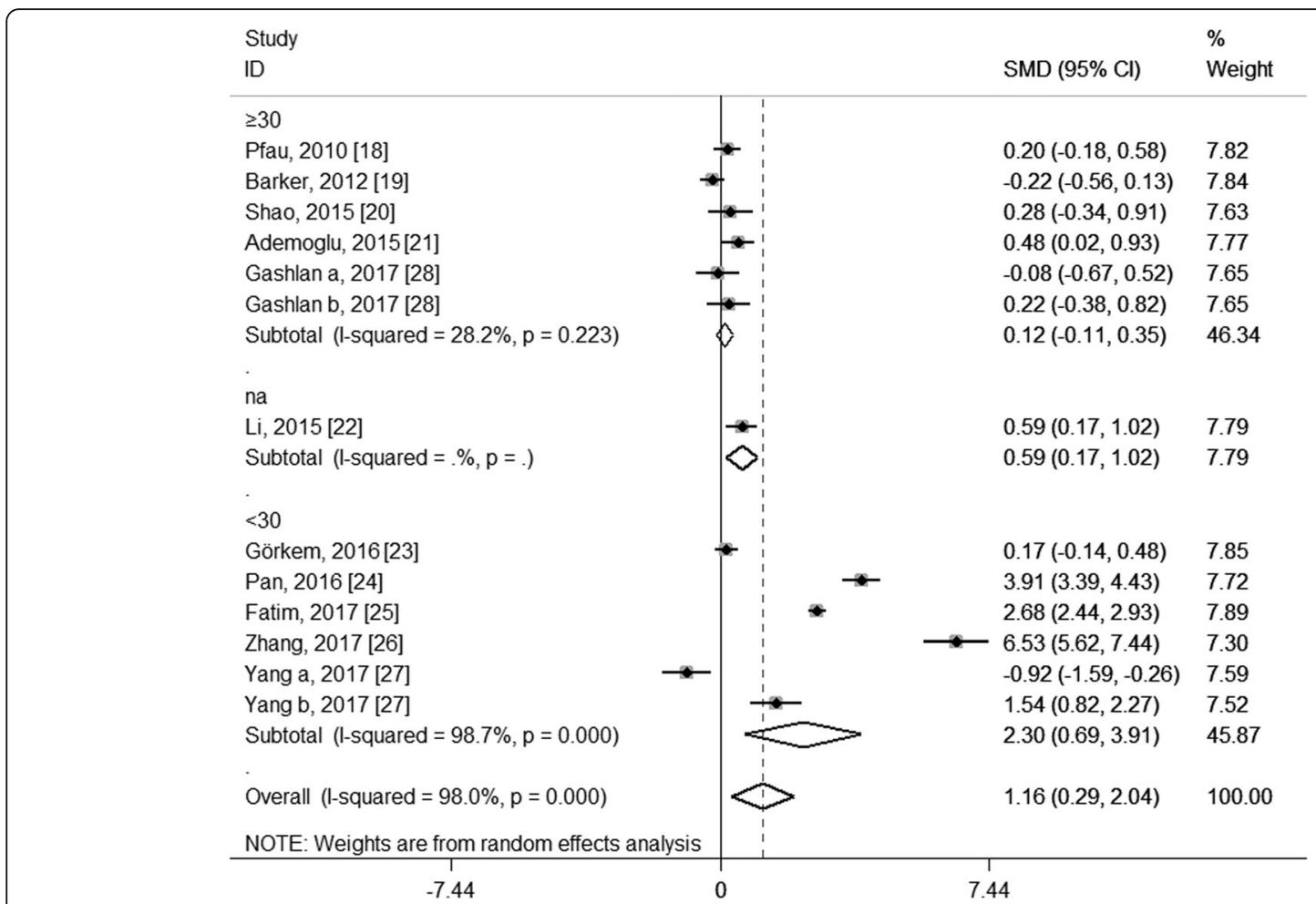

Fig. 6 Subgroup analysis of circulating chemerin levels in gestational diabetes mellitus (GDM) patients compared with healthy pregnant women when stratified by the average age of GDM patients. SMD, standardized mean differences; Cl, confidence interval; na, not available

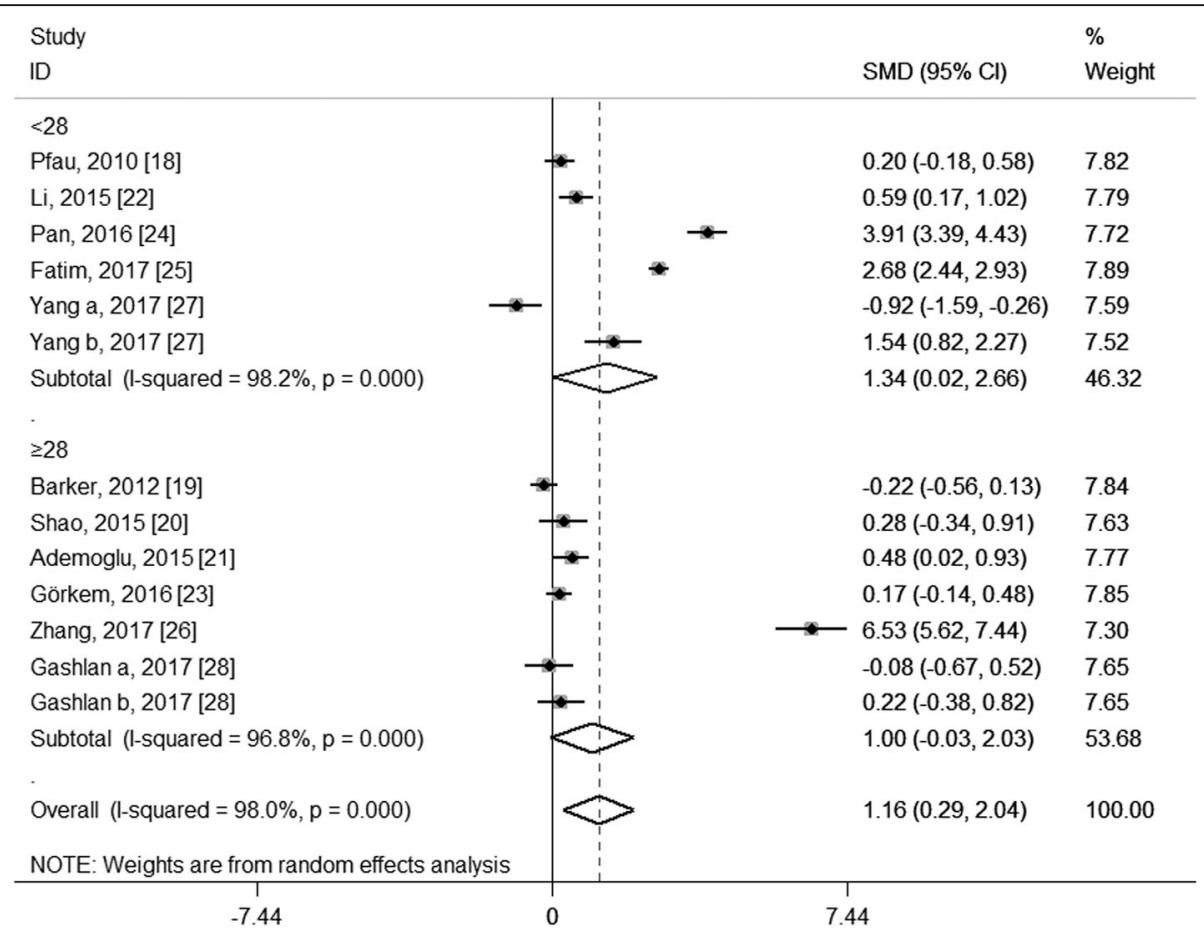

Fig. 7 Subgroup analysis of circulating chemerin levels in gestational diabetes mellitus (GDM) patients compared with healthy pregnant women when stratified by mean body mass index of GDM patients. SMD, standardized mean differences; Cl, confidence interval 


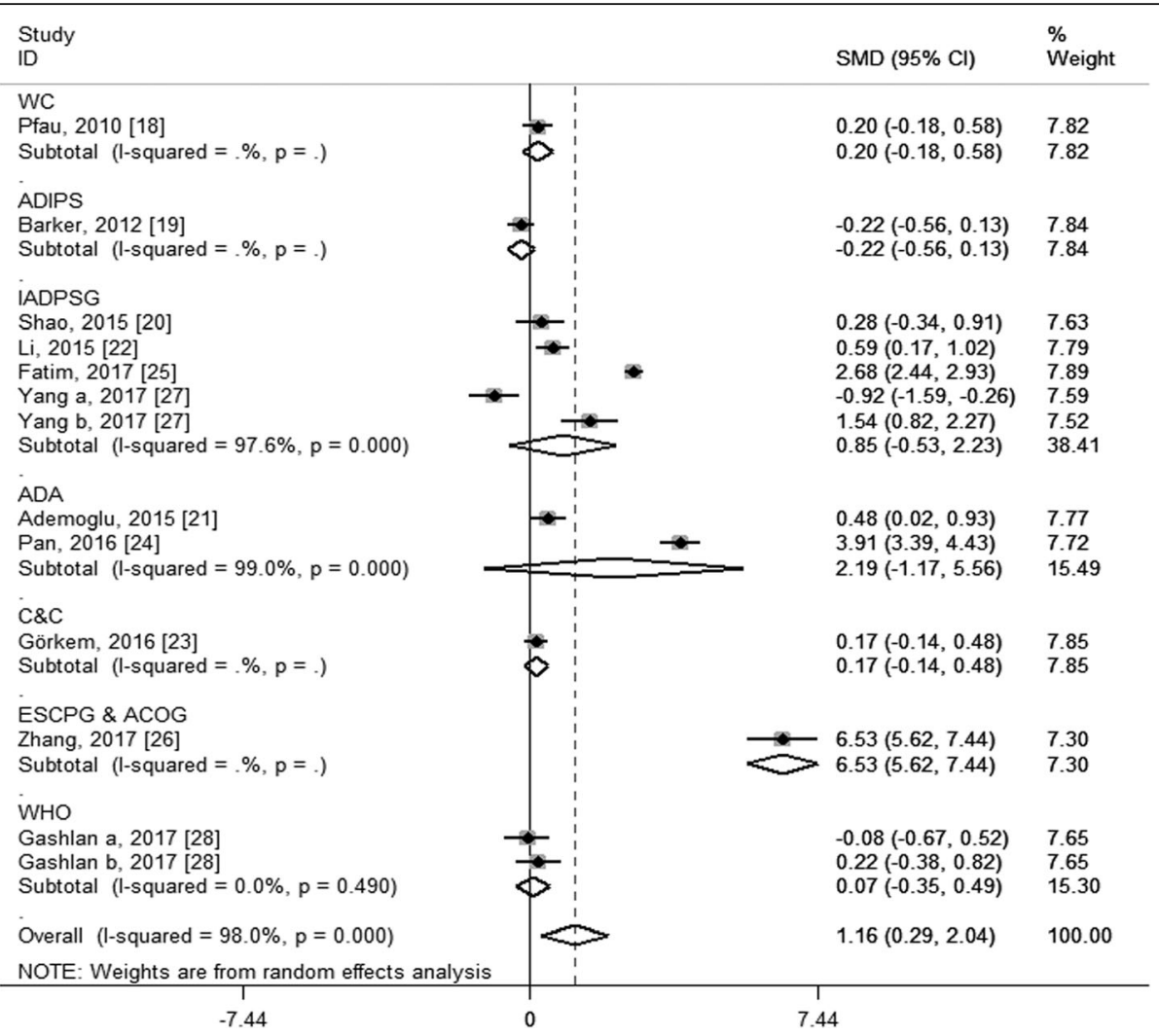

Fig. 8 Subgroup analysis of circulating chemerin levels in gestational diabetes mellitus (GDM) patients compared with healthy pregnant women when stratified by the diagnostic criteria of GDM. WC, Fourth International Work-shopVconference on gestational diabetes; ADIPS, The Australasian Diabetes in Pregnancy Society; IADPSG, The International Association of Diabetes and Pregnancy Study Groups; ADA, American Diabetes Association; C\&C: Carpenter and Couston; ESCPG, Endocrine Society Clinical Practice Guideline; ACOG, American College of Obstetricians and Gynecologists; WHO, World Health Organization; SMD, standardized mean differences; Cl, confidence interval

to confirm their link in GDM patients. In this meta-analysis, we did not find higher levels of circulating chemerin in higher BMI group, although previous research has indicated that elevated chemerin expression was associated with obesity and obesity-related disease. The possible explanation is that BMI is not an ideal index for reflecting obesity, as it balances both height and weight that reflect the total body fat. However, the indexes reflecting central obesity such as waist circumference and waist-hip ratio cannot be acquired in studies included in this meta-analysis.

In recent years, an increasing number of studies have found that a variety of adipokines may be involved in the pathophysiology of GDM. A review in 2014 by Fasshauer et al. [32] provided an overview of various adipokines in GDM, and suggested adiponectin, leptin, tumour necrosis factor $\alpha(T N F \alpha)$ and adipocyte fatty acid-binding protein (AFABP) are more likely than chemerin and other adipokines to play roles in the pathogenic mechanism of GDM based on previous reports. In this paper, we collected 11 studies which were systematically and quantitatively analyzed and provided a more comprehensive estimation that chemerin may play a powerful role in the pathophysiology of GDM. The mechanism that chemerin involves in endocrine and metabolic regulation is highly intricate because of its several isoforms, differential expression in different organs and the diverse activation mechanism [8, 33 32]. As the pathophysiologic mechanism of GDM is similar to T2DM in which insulin resistance and chronic inflammation are the most important pathophysiological basis, and elevated chemerin expression has been shown to contribute to the development of insulin resistance and low-grade chronic inflammation [8-10], it is possible that chemerin involved in the pathophysiologic mechanism of GDM is by increasing insulin resistance and promoting subclinical inflammation. Several studies included this meta-analysis also indicated that GDM patients had significantly higher levels of homoeostasis model assessment of insulin resistance (HOMA-IR) and inflammatory parameters such as $\mathrm{C}$-reactive protein when compared with healthy pregnant women [22-26], and there were significant positive correlations between serum chemerin levels and insulin resistance, and inflammatory parameters [18, 22, 24]. 
There are several limitations in this met-analysis. First, remarkable heterogeneity among studies limits the reliability of the results. Although subgroup analyses were used to explore the potential sources, the high levels of heterogeneity cannot be sufficiently and reasonably explained. Moreover, as data on some potential confounder such as exercise, diet adjustment and the levels of inflammation and insulin resistance are limited in the eligible studies included in the meta-analysis, we cannot analyze whether these factors had moderating effects on the pooled results. Therefore, the results of this meta-analysis should be cautiously interpreted. Second, all of the studies included in this meta-analysis were cross-sectional, thus a causal relationship between circulating chemerin levels and GDM cannot be established. Third, the publication bias may not be avoided absolutely although Egger's test was applied in this analysis, as only published studies in English and Chinese in the selected databases were included. Finally, the quality assessment of studies included in this meta-analysis was based on the modified NOS due to the lack of appropriate assessment tool for cross-sectional studies, which might result in arbitrary results [33, 34].

\section{Conclusions}

In conclusion, this meta-analysis demonstrated that the elevated levels of circulating chemerin were associated with GDM, which suggests it might play an important role in the pathogenetic mechanism of GDM. However, the results should be cautiously interpreted owing to substantial heterogeneity among studies, and further prospective cohort studies are required to confirm these findings.

\section{Funding}

All the authors received no financial support in this work.

\section{Availability of data and materials}

The datasets used and analyzed during the current study are available from the corresponding author on reasonable request.

\section{Authors' contributions}

ZZ and HC contributed to literature search, data extraction, quality assessment and drafted the manuscript. HJ contributed to editing and revision of the manuscript. MS designed the study, and performed data interpretation, revision of the manuscript. All authors read and approved the final manuscript.

\section{Ethics approval and consent to participate}

Not applicable.

\section{Consent for publication}

Not applicable.

\section{Competing interests}

The authors declare that they have no competing interests.

\section{Publisher's Note}

Springer Nature remains neutral with regard to jurisdictional claims in published maps and institutional affiliations.
Received: 30 January 2018 Accepted: 11 July 2018

Published online: 24 July 2018

\section{References}

1. Simmons D. Prevention of gestational diabetes mellitus: where are we now? Diabetes Obes Metab. 2015;17:824-34.

2. Cundy T, Ackermann E, Ryan EA. Gestational diabetes: new criteria may triple the prevalence but effect on outcomes is unclear. BMJ. 2014;348: g1567.

3. Allalou A, Nalla A, Prentice KJ, Liu Y, Zhang M, Dai FF, Ning X, Osborne $L R$, Cox BJ, Gunderson EP, Wheeler MB. A predictive metabolic signature for the transition from gestational diabetes mellitus to type 2 diabetes. Diabetes. 2016;65:2529-39.

4. Wang YH, Wu HH, Ding H, Li Y, Wang ZH, Li F, Zhang JP. Changes of insulin resistance and beta-cell function in women with gestational diabetes mellitus and normal pregnant women during mid- and late pregnant period: a case-control study. J Obstet Gynaecol Res. 2013;39:647-52.

5. Zhang H, Zhang J, Pope CF, Crawford LA, Vasavada RC, Jagasia SM, Gannon M. Gestational diabetes mellitus resulting from impaired betacell compensation in the absence of FoxM1, a novel downstream effector of placental lactogen. Diabetes. 2010;59:143-52.

6. Nordmann TM, Dror E, Schulze F, Traub S, Berishvili E, Barbieux C, BoniSchnetzler M, Donath MY. The role of inflammation in beta-cell dedifferentiation. Sci Rep. 2017;7:6285.

7. Lekva T, Norwitz ER, Aukrust P, Ueland T. Impact of systemic inflammation on the progression of gestational diabetes mellitus. Curr Diab Rep. 2016;16:26.

8. Fatima SS, Rehman R, Baig M, Khan TA. New roles of the multidimensional adipokine: chemerin. Peptides. 2014;62:15-20.

9. Bondue B, Wittamer V, Parmentier M. Chemerin and its receptors in leukocyte trafficking, inflammation and metabolism. Cytokine Growth Factor Rev. 2011;22:331-8.

10. Yilmaz Y, Yonal O, Kurt R, Alahdab YO, Eren F, Ozdogan O, Celikel CA, Imeryuz N, Kalayci C, Avsar E. Serum levels of omentin, chemerin and adipsin in patients with biopsy-proven nonalcoholic fatty liver disease. Scand J Gastroenterol. 2011;46:91-7.

11. Docke S, Lock JF, Birkenfeld AL, Hoppe S, Lieske S, Rieger A, Raschzok N, Sauer IM, Florian S, Osterhoff MA, Heller R, Herrmann K, Lindenmuller S, Horn P, Bauer M, Weickert MO, Neuhaus P, Stockmann M, Mohlig M, Pfeiffer $A F$, von Loeffelholz C. Elevated hepatic chemerin mRNA expression in human non-alcoholic fatty liver disease. Eur J Endocrinol. 2013;169:547-57.

12. Zylla S, Pietzner M, Kuhn JP, Volzke H, Dorr M, Nauck M, Friedrich N. Serum chemerin is associated with inflammatory and metabolic parameters-results of a population-based study. Obesity (Silver Spring). 2017:25:468-75.

13. Bobbert T, Schwarz F, Fischer-Rosinsky A, Maurer L, Mohlig M, Pfeiffer AF, Mai K, Spranger J. Chemerin and prediction of diabetes mellitus type 2. Clin Endocrinol. 2015:82:838-43.

14. Leiherer A, Muendlein A, Kinz E, Vonbank A, Rein P, Fraunberger $P$, Malin C, Saely $\mathrm{CH}$, Drexel $\mathrm{H}$. High plasma chemerin is associated with renal dysfunction and predictive for cardiovascular events - insights from phenotype and genotype characterization. Vasc Pharmacol. 2016;77:60-8.

15. van Dijk GM, Maneva M, Colpani V, Dhana K, Muka T, Jaspers L, Kavousi M, Franco $\mathrm{OH}$. The association between vasomotor symptoms and metabolic health in peri- and postmenopausal women: a systematic review. Maturitas. 2015:80:140-7.

16. Masi A, Quintana DS, Glozier N, Lloyd AR, Hickie IB, Guastella AJ. Cytokine aberrations in autism spectrum disorder: a systematic review and metaanalysis. Mol Psychiatry. 2015;20:440-6.

17. Ioannidis JP, Patsopoulos NA, Evangelou E. Uncertainty in heterogeneity estimates in meta-analyses. BMJ. 2007;335:914-6.

18. Pfau D, Stepan H, Kratzsch J, Verlohren M, Verlohren HJ, Drynda K, Lossner U, Bluher M, Stumvoll M, Fasshauer M. Circulating levels of the adipokine chemerin in gestational diabetes mellitus. Horm Res Paediatr. 2010;74:56-61.

19. Barker G, Lim R, Rice GE, Lappas M. Increased chemerin concentrations in fetuses of obese mothers and correlation with maternal insulin sensitivity. J Matern Fetal Neonatal Med. 2012;25:2274-80.

20. Shao J, Liu M, Li F, Xu X. The expression of Chemerin in maternal peripheral, umbilical cord blood and placenta in gestational diabetic patients. J Biomater Tis Eng. 2015;5:833-7. 
21. Ademoglu E, Berberoglu Z, Dellal FD, Ariel MK, Kose A, Candan Z, Bekdemir $\mathrm{H}$, Erdamar $\mathrm{H}$, Culha C, Aral Y. Higher levels of circulating chemerin in obese women with gestational diabetes mellitus. Acta Endocrinol. 2015;11:32-7.

22. Li XM, Ji H, Li CJ, Wang PH, Yu P, Yu DM. Chemerin expression in Chinese pregnant women with and without gestational diabetes mellitus. Ann Endocrinol. 2015;76:19-24.

23. Gorkem U, Kucukler FK, Togrul C, Gungor T. Are adipokines associated with gestational diabetes mellitus? J Turk Ger Gynecol Assoc. 2016;17:186-90.

24. Pan BL, Ma RM. Correlation of serum omentin-1 and chemerin with gestational diabetes mellitus. Nan Fang Yi Ke Da Xue Xue Bao. 2016;36: $1231-6$.

25. Fatima SS, Alam F, Chaudhry B, Khan TA. Elevated levels of chemerin, leptin, and interleukin-18 in gestational diabetes mellitus. J Matern Fetal Neonatal Med. 2017;30:1023-8.

26. Zhang J, Chi H, Xiao H, Tian X, Wang Y, Yun X, Xu Y. Interleukin 6 (IL-6) and tumor necrosis factor a (TNF-a) single nucleotide polymorphisms (SNPs), inflammation and metabolism in gestational diabetes mellitus in Inner Mongolia. Med Sci Monit. 2017;23:4149-57.

27. Yang X, Quan X, Lan Y, Ye J, Wei Q, Yin X, Fan F, Xing H. Serum chemerin level during the first trimester of pregnancy and the risk of gestational diabetes mellitus. Gynecol Endocrinol. 2017;33:770-3.

28. Gashlan HM. Relationship between levels of retinol binding protein 4 Vaspin and Chemerin and insulin resistance in gestational diabetes mellitus. Int J Pharmaceut Res All Sc. 2017;6:236-50

29. Svensson H, Oden B, Eden S, Lonn M. Adiponectin, chemerin, cytokines, and dipeptidyl peptidase 4 are released from human adipose tissue in a depotdependent manner: an in vitro system including human serum albumin. BMC Endocr Disord. 2014;14:7.

30. Cheon DY, Kang JG, Lee SJ, Ihm SH, Lee EJ, Choi MG, Yoo HJ, Kim CS. Serum Chemerin levels are associated with visceral adiposity, independent of waist circumference, in newly diagnosed type 2 diabetic subjects. Yonsei Med J. 2017;58:319-25

31. Motawi TMK, Mahdy SG, El-Sawalhi MM, Ali EN, El-Telbany RFA. Serum levels of chemerin, apelin, vaspin, and omentin-1 in obese type 2 diabetic Egyptian patients with coronary artery stenosis. Can J Physiol Pharmacol. 2017:1-7.

32. Fasshauer M, Blüher M, Stumvoll M. Adipokines in gestational diabetes. Lancet Diabetes Endocrinol. 2014;2:488-99.

33. Mattern A, Zellmann T, Beck-Sickinger AG. Processing, signaling, and physiological function of chemerin. IUBMB Life. 2014;66:19-26.

34. Stang A. Critical evaluation of the Newcastle-Ottawa scale for the assessment of the quality of nonrandomized studies in meta-analyses. Eur J Epidemiol. 2010;25:603-5.

Ready to submit your research? Choose BMC and benefit from:

- fast, convenient online submission

- thorough peer review by experienced researchers in your field

- rapid publication on acceptance

- support for research data, including large and complex data types

- gold Open Access which fosters wider collaboration and increased citations

- maximum visibility for your research: over $100 \mathrm{M}$ website views per year

At $\mathrm{BMC}$, research is always in progress.

Learn more biomedcentral.com/submissions 